\title{
Cyclic Prefix Optimization of OFDM System
}

\author{
Shashikant ${ }^{1}$, Divya Dhawan ${ }^{2}$ \\ ${ }^{I}$ (ECE Department, PEC Chandigarh, India)
}

\begin{abstract}
There are many methods to reduce the multipath effects in wireless communication. Multicarrier modulation for data transmission is one of the methods to overcome the effect of Inter Symbol Interference (ISI), also known as Orthogonal Frequency Division Multiplexing (OFDM). The analysis of Bit Error Rate (BER) performance suggests, OFDM is better than Code Division Multiple Access (CDMA) which is mostly incorporated in existing $3 G$ systems. [1]In this article the influence of cyclic prefix and pilot insertion technique on an OFDM system is analyzed on an AWGN channel. The OFDM system is simulated with MATLAB programming as well as simulink model, and the BER curves with and without CP/PI are compared. The variance of power loss and information rate loss with length of the cyclic prefix is explained.
\end{abstract}

Keywords: BER, CP , FFT,OFDM,PI, Simulink.

\section{INTRODUCTION}

The objective of OFDM is to separate the wide frequency selectivity of fading channels into multiple flat fading channels. Instead of using analog subcarrier oscillators Discrete Fourier Transform (DFT) technique is used to generate and receive OFDM symbols. The key to OFDM is maintaining orthogonality of the carriers. If the integral of the product of two signals is zero over a time period, then these two signals are said to be orthogonal to each other. Two sinusoids with frequencies that are integer multiples of a common frequency can satisfy this criterion. Therefore, orthogonality is defined by:

$$
\int_{0}^{T} \cos (2 \pi n f o t) \cos (2 \pi m f o t) d t=0, \quad(n \neq m)
$$

where $\mathrm{n}$ and $\mathrm{m}$ are two unequal integers; fo is the fundamental frequency; $\mathrm{T}$ is the period over which the integration is taken.[2] The various symbols are transmitted at orthogonal frequencies so that interference due to their multiplication part becomes zero. An OFDM signal is a sum of subcarriers that are individually modulated by using phase shift keying (PSK) or quadrature amplitude modulation (QAM). The symbol can be written as:

$$
\mathrm{x}[\mathrm{n}]=\sum_{k=0}^{N-1} \mathrm{x}[\mathrm{k}] e^{j 2 \pi n / N} \text { for } \mathrm{n}=0,1, \ldots \ldots, \mathrm{N}-1
$$

The above equation can be computed efficiently by using N-point IFFT algorithm[4]. Let $y[n]$ is the received signal then OFDM symbols can be recovered by using as follows :

$$
\mathrm{y}[\mathrm{k}]=\sum_{n=0}^{N-1} \mathrm{y}[\mathrm{n}] e^{-j 2 \pi n / N}
$$

The above equation (3) can be easily calculated by using FFT algorithm. So to implement the OFDM technique IFFT and FFT algorithms are used in transmitter and receiver side respectively.

OFDM system performance can be improved by adding pilot signals and cyclic prefix to the transmitting signal. In this article basic principle of OFDM is briefly introduced, and on this basis the simulation model of OFDM is built, then by way of $\mathrm{CP}$ and PI the performance of OFDM system is analyzed.

1.1 PILOT synchronization technique: This technique is used in channel estimation in wireless channels which are time variant and needs to be tracked continuously, where a pilot symbol is inserted in the OFDM signal at specified locations and the same are then tracked on the receiver side and eliminated. These pilot symbols acts as marking flags in the AWGN signal and can be identified with less interference thus reducing BER [3].

1.2 Cyclic prefix: ISI in OFDM system system can be reduced by using guard intervals of null value but that will disrupt the orthogonality of the subcarriers. So guard space filled with cyclic prefix is used where the last few samples are added to the front of the signal. The symbol period increases from $\mathrm{T}$ to $\mathrm{T}+\mathrm{dT}$, dT is the guard interval kept less than or equal to $\mathrm{T} / 4$, increasing further reduces the frequency spectrum utilization[5]. 


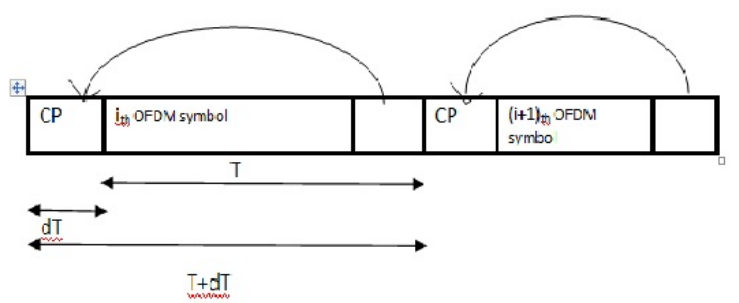

Fig.1 Cyclic Prefix

Information rate reduces and power is lost when $\mathrm{CP}$ is inserted as:

$v=10 \log \left(\frac{T_{g}}{T}+1\right)$

Where $\mathrm{Tg}$ is the cyclic guard interval and $\mathrm{T}$ data symbol time duration.

Addition of cyclic bits leads to the information rate loss as these bits do not carry any information regarding the transmitting message. So more is the information rate which limits the length of cyclic prefix. This article's focus is on optimizing the length of cyclic prefix in an OFDM system.

$$
\text { Information rate loss }=\frac{\text { cyclic }_{\text {Symbols }}}{\text { Total }_{\text {symbols }}} * 100
$$

II. BLOCK DIAGRAM

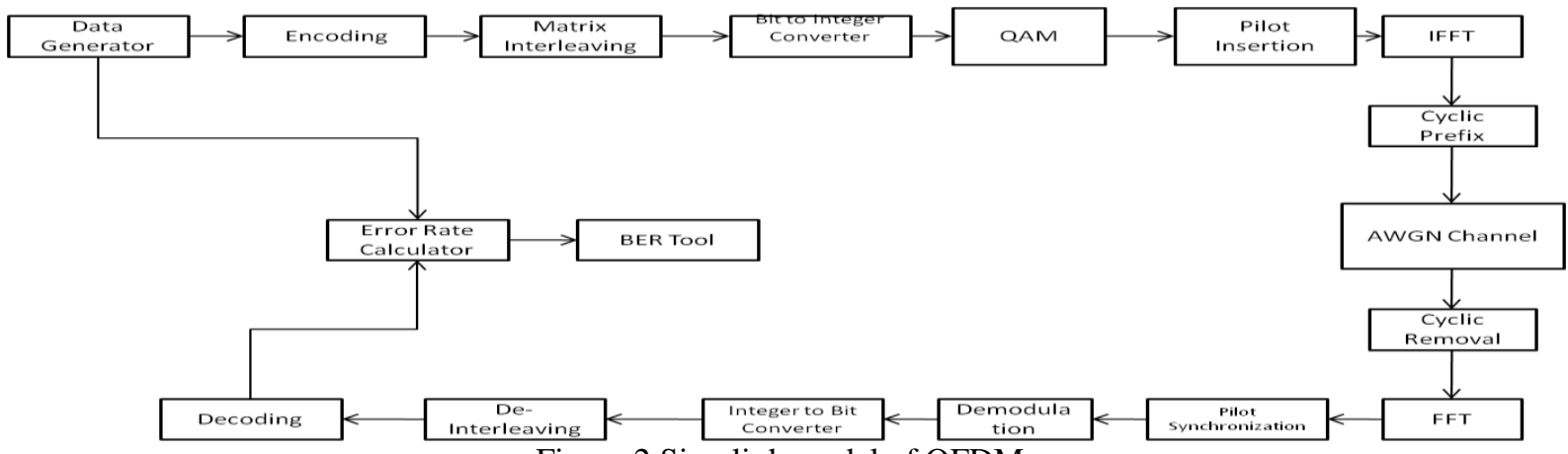

Figure 2 Simulink model of OFDM

The block diagram of the system is shown in Fig.2. In this model cyclic prefix and pilot insertion techniques are implemented with programmed CP and PI blocks on transmitter as well as receiver side. Binary data is first encoded using a convolution encoder. Encoded data is then converted into integer form for QAM modulation after interleaving. QAM modulated data is then implemented with Pilot insertion which also includes resizing of the data matrix. After inverse Fast Fourier Transform (IFFT) of PI data, Cyclic prefix are added and then transmitted on an AWGN channel. On receiver side CP is first removed and then FFT of that data matrix is passed for Pilot synchronization which further is demodulated.

The demodulated data is then converted in binary form and decoded after deinterleaving. Error rate is calculated and BER curve is plotted [6].

Table 1. List of Parameters

\begin{tabular}{|l|l|}
\hline Number of frames sent & 100 \\
\hline No. of samples per frame & 96 \\
\hline No. of Subcarriers & 64 \\
\hline No. of Pilots & 4 \\
\hline Modulation & 16 \\
\hline Interleaving & $48 \times 4$ \\
\hline
\end{tabular}

\section{SimUlation ANALYSIS}

Fig.3 shows the performance of the system without using Cyclic prefix/pilot synchronization techniques .When SNR is increasing BER decreases but at $10 \mathrm{db}$ the BER starts increasing and then fall back at $12 \mathrm{db}$. BER shows same pattern between $15 \mathrm{db}$ to $17 \mathrm{db}$ and $22 \mathrm{db}$ to $25 \mathrm{db}$.

Fig. 5 shows the performance of the system using Cyclic prefix/pilot synchronization technique. 
Curve shows that BER decreases very smoothly with increasing SNR and shows no spikes along the curve as seen in Fig.3.

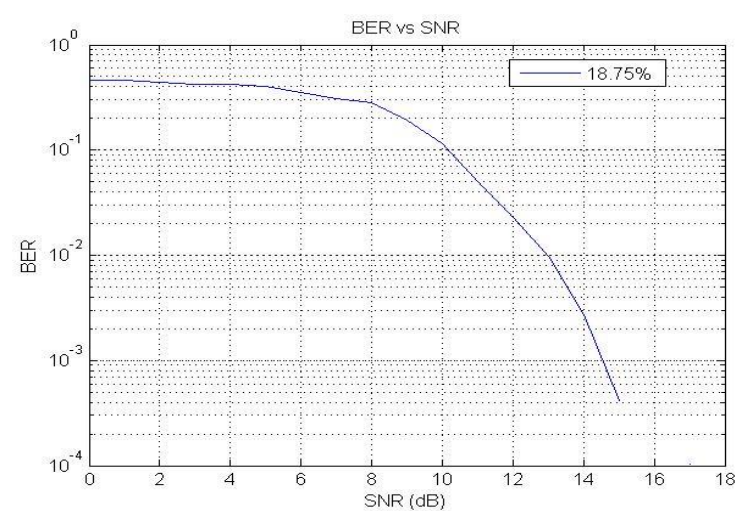

Figure 3. BERvs SNR with $\mathrm{CP}=18.75 \%$

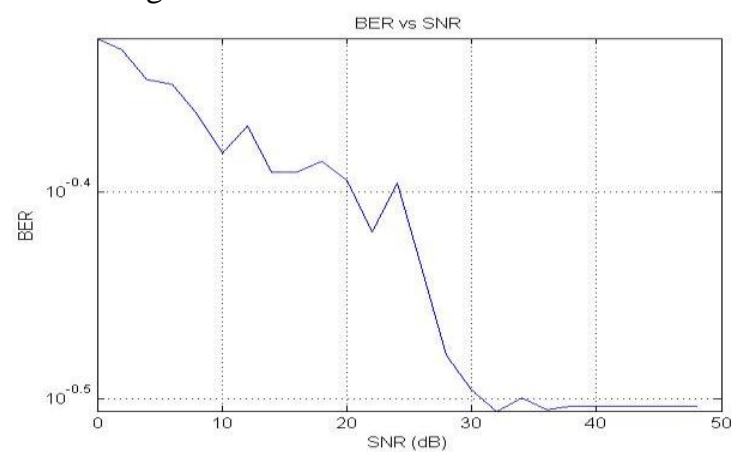

Figure 4. BER vs SNR with NO CP

Also with BER value of $10^{\wedge}-.5$ even above $30 \mathrm{~dB}$ in Fig.3, BER value of above $10^{\wedge}-3$ is achieved that even at 14dB SNR using CP and PI technique as shown in Fig.6 thus reducing ISI to greater extent.

Table 2 shows the various values of information rate loss and power loss with the change in cyclic extension. Fig.7 shows the variation of power loss (dB) with respect to cyclic extensions . The variance is described in the equation (2) which is a logarithmic graph. The relation between Information rate loss with respect to cyclic extension is shown in Fig. 7 which resembles with the relation described in equation (4).

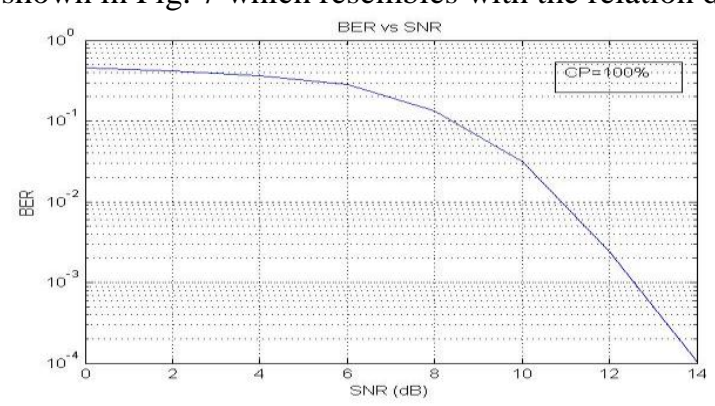

Figure 5. BER vs SNR wih CP $100 \%$

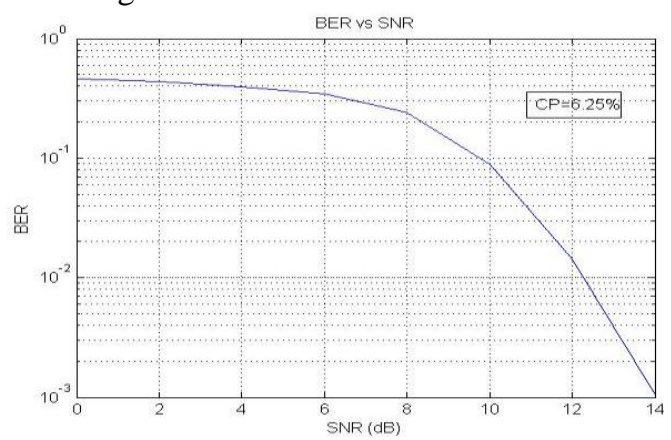

Figure 6. BER vs SNR with $\mathrm{CP}=6.25 \%$ 
As the cyclic prefix length is increased more than 18.75 percent the power loss becomes more than 1 $\mathrm{dB}$ but till then BER value of .0005 is achieved as shown in Fig.5 With 100 percent cyclic prefix, BER value of .0001 is achieved below 14dB SNR but at the cost of $3 \mathrm{~dB}$ power loss as shown in Table.1 which is not efficient. Information rate loss varies vigorously with cyclic extension as seen in Fig.8. With 25 percent CP IR loss is 20 percent and 6.25 percent CP the IR loss reduces to 6 percent with BER .001 as in fig. 5. Greater the IR loss more is the requirement of bandwidth.

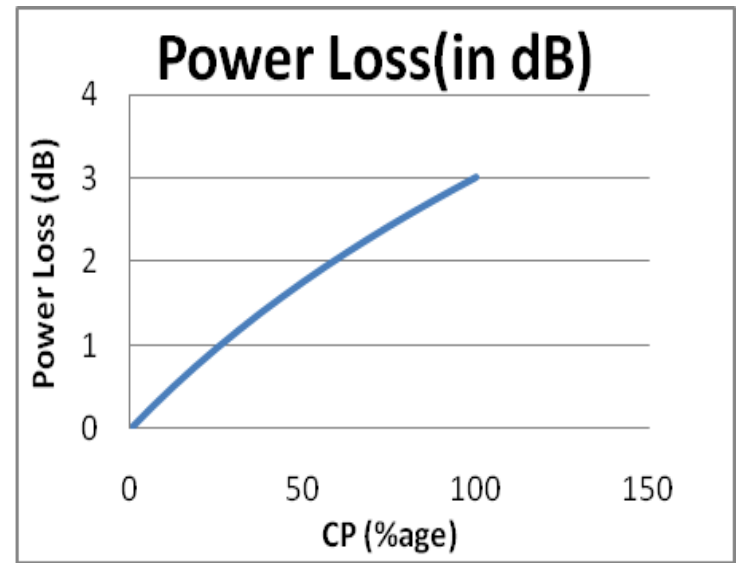

Figure 7 Power Loss vs CP

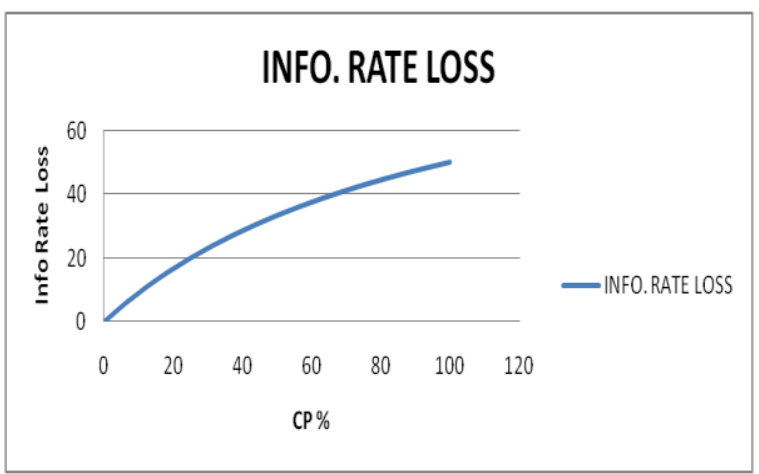

Fig.8.Information Rate Loss vs CP

\section{CONCLUSION}

Cyclic prefix and pilot synchronization improves the OFDM system in terms of BER of the system which also depends on the length of the cyclic prefix .Till a certain value of Cyclic Prefix(20 percent), BER value decreases which further remains constant (.0001). Higher value of BER is achieved at the cost of information rate loss which increases rapidly with CP. Bandwidth of the system limits the extension in Cyclic Prefix that concludes that in order to design an efficient system one has to optimize these three parameters CP, power loss and bandwidth.

\section{REFERENCES}

[1]. Jigisha N. Patel „Prof.(Mrs.) Upena D.Dalal, A Comparative Performance Analysis of OFDM using MATLAB Simulation with M-PSK and M-QAM Mapping, International Conference on Computational Intelligence and Multimedia Applications 2007.

[2]. Xiurong Bao, Matlab Simulation and Performance Analysis of OFDM System, International Conference on Computer Science and Information Processing (CSIP),2012.

[3]. Sinem Coleri, Mustafa Ergen, Anuj Puri, and Ahmad Bahai, Channel Estimation Techniques Based on Pilot Arrangement in OFDM Systems ,IEEE TRANSACTIONS ON BROADCASTING, VOL. 48, NO. 3, SEPTEMBER 2002.

[4]. Mrs.S.S.GHORPADE, Prof.Dr.Mrs.S.V.SANKPAL , Behavior of OFDM System Using MATLABSimulation , INTERNATIONAL JOURNAL OF INNOVATIVE TECHNOLOGY AND RESEARCH Volume No. 1, Issue No. 3, April - May $2013,249-252$.

[5]. Miroslav Makarov, MATLab simulation for Comparison of Different QAM Constellations and Mapping for a Chanel with AWGN , Conference of Informatics and Management Sciences ,March, 25-29 2013.

[6]. Orlandos grigoriadis, H.Srikanth kamath, BER calculation using matlab simulation for ofdm transmission, Proceedings of the International Multi Conference of Engineers and Computer Scientists,2008 Vol II, IMECS 2008, 19-21 March, 2008, Hong Kong. 